\title{
The Initiation Phase of Warfarin Therapy: Differences of Dose among Patients with Aortic Valve Replacement, Mitral Valve Replacement and Atrial Fibrillation
}

\author{
Teoh Chee Jia ${ }^{1}$, Aniza Abd Aziz ${ }^{2}$, Nadiah Wan-Arfah ${ }^{1}$, Nyi Nyi Naing ${ }^{1 *}$ \\ ${ }^{1}$ Unit of Biostatistics and Research Methodology, School of Medical Sciences, Universiti Sains Malaysia, 16150 Kubang Kerian, Kelantan, Malaysia. \\ ${ }^{2}$ Faculty of Medicine, Universiti Sultan Zainal Abidin, Jalan Sultan Mahmud, 20400 Kuala Terengganu, Terengganu, Malaysia.
}

\section{ARTICLE INFO \\ Article history: \\ Received on: 01/06/2016 \\ Revised on: 18/08/2016 \\ Accepted on: 05/09/2016 \\ Available online: $29 / 11 / 2016$}

Key words:

Warfarin; international

normalized ratio; atrial

fibrillation.

\begin{abstract}
This study was conducted to evaluate the changes in warfarin doses among patients after heart valve surgery and atrial fibrillation. A total of 137 patients having AF, AVR and MVR whom initiate warfarin therapy in year 2008 till 2010 in Penang Hospital were selected as samples for this study. Data collected included patient's age, race, gender, warfarin initiation date, warfarin indication, warfarin dose and INR value for $1^{\text {st }}$ till $12^{\text {th }}$ weeks of warfarin initiation. Heart valve replacement patients (AVR or MVR) showed increasing trend in warfarin doses during the initial phase of warfarin therapy. This trend was not observed in AF patients. The dose of warfarin was significantly different within first 12 weeks of warfarin initiation for AVR and MVR group $(p<0.001)$ but there was no significant difference in AF group. The dose of warfarin was significantly different among AVR and AF patients for first 5 weeks of warfarin initiation. Monitoring should be frequent for patients with heart valve replacement during the initial 3 months period of warfarin therapy.
\end{abstract}

\section{INTRODUCTION}

Warfarin, an oral anticoagulant, is widely used for preventing and treating vascular and thromboembolic disease (Haines et al., 2008). Almost 20 million prescriptions are written for warfarin each year in the US and it is one of the most challenging drugs in the modern medical formulary (Jonas and McLeod, 2009). In Malaysia, warfarin remained the mainstay of the Vitamin $\mathrm{K}$ antagonist group, with about $0.05 \%$ of the population in Malaysia used warfarin everyday in a year (Sameerah and Sarojini, 2007). The therapeutic index of warfarin is narrow in any patient (Haines et al., 2008) and patient's response to warfarin is highly variable. The dose requirement of warfarin can vary more than 10-fold between patients (Hall and Wilkins, 2005; Takahashi et al., 2003). Age, weight, dietary

* Corresponding Author

Nyi Nyi Naing, Unit of Biostatistics and Research Methodology, School of Medical Sciences, Universiti Sains Malaysia, 16150 Kubang Kerian, Kelantan, Malaysia.Email: nyi @ usm.my intake of vitamin $\mathrm{K}$, concurrent medication, gender, liver disease, congestive heart failure, serum albumin and Cytochrome P450 polymorphism, alcohol intake and patient's compliance contributed to the variation of the warfarin dose among patients (Hall and Wilkins, 2005; Lee et al., 2005; Wadelius et al., 2004). The period of highest risk of bleeding complications is around the initiation of warfarin treatment (Douketis et al., 2000; Kulik et al., 2006). The risk of thromboembolic events is higher in the early (< 3 months) versus late postoperative phase. The challenge of initiation of warfarin will be to balance the risks of underanticoagulation (thromboembolic events) against those of overanticoagulation (bleeding complications), when the risk of bleeding may be significant in the initiation of treatment (Kulik et al., 2006). Lowest adequate intensity of anticoagulation is important to minimize the risk of bleeding (Fuster et al., 2001). The sensitivity to warfarin decrease after the initial period and the progressive decrease in warfarin sensitivity over several months often cause difficulty to reach and maintain a stable INR within therapeutic range (Meijer et al., 2009; Rahman et al., 2006). 
This study looked into the difference in warfarin doses after the initiation of warfarin therapy among patients with aortic valve replacement (AVR), mitral valve replacement (MVR) or atrial fibrillation (AF) and also between these three groups. To our knowledge, similar study has not been carried out in Malaysia. Studies from Western countries and north Asia countries may not be applicable to Malaysian population due to differences in geographical area and ethnicity. Malaysia is a multiracial and multicultural country. Difference in races and lifestyle were known to be influential in warfarin dosing. The aim of the study was to compare the warfarin doses differences in initial phase of warfarin therapy among patients with aortic valve replacement, mitral valve replacement and atrial fibrillation.

\section{MATERIAL AND METHODS}

\section{Study Design and Participants}

A retrospective record review study was conducted at warfarin clinic of Hospital Penang. The warfarin clinic was established in year 2008 for the patients from cardiothoracic and cardiology clinic. Patients from Cardiothoracic and Cardiology clinic who are on warfarin therapy were referred to this clinic for review and management. The source population was patients having atrial fibrillation, aortic valve replacement and mitral valve replacement who initiate warfarin therapy in year 2008 till 2010 in Penang Hospital. The sampling frame was patients having atrial fibrillation, aortic valve replacement and mitral valve replacement who initiated warfarin therapy in year 2008 till 2010 in Penang Hospital who fulfilled inclusion and exclusion criteria.

Patient who had INR within therapeutic range and who was having warfarin treatment after heart valve surgery or atrial fibrillation and follow up full 3 months in Penang General Hospital after heart valve surgery or atrial fibrillation were the inclusion criteria (Salem et al., 2008). The exclusion criteria were atrial fibrillation patient who had undergone cardiac surgery 3 months before starting warfarin and patient having double valve replacement (Meijer et al., 2009).

\section{Case Definitions}

Warfarin dose was defined as dose of warfarin in mg per day. Initial phase of warfarin therapy was defined as the first three months from starting the warfarin therapy. Dose of warfarin at Week 1 was defined as dose of warfarin in mg per day at the last day of the first week after patient's initiation of warfarin therapy. Data collected included patient's age, race, gender, warfarin initiation date, warfarin indication, INR value and twelve readings of warfarin dose for $1^{\text {st }}$ till $12^{\text {th }}$ weeks of warfarin initiation.

\section{Statistical Analysis}

All statistical analysis was performed using SPSS software version 17. Baseline characteristics were presented in frequencies and percentages or were expressed as mean and standard deviation. These included indication, age, race and gender. One-way repeated measures ANOVA and multi-way repeated measures ANCOVA analysis was used to evaluate the significance of difference between groups and among groups. Three types of effect were analyzed using one-way repeated measures ANOVA model. These effects were within warfarin indication group (Time effect), between warfarin indication groups (Group effect) and within-between design (Time-Group Interaction). Multi-way repeated measures ANCOVA analysis was used to analyze between warfarin indication groups effect (Group effect) and within-between design (Time-Group Interaction) after controlled age, gender and race. Three assumptions were tested for one-way repeated measures ANOVA and multi-way repeated measures ANCOVA analysis. First, the assumption of normality was tested. All 12 residuals were checked by using histogram and box and whisker plot. Second, the assumption of homogeneity of variances was tested by using Levene's test of Equality of Error Variances and observations in the scatter plots of all twelve residual vs predicted values. Third, the assumption of compound symmetry was tested by using Mauchly's test of sphericity. Results were considered significant at a $95 \%$ level of confidence.

\section{Ethical Approval}

The study received approval from The Research Ethics Committee (Human) of Universiti Sains Malaysia (USMKK/PPP/JEPeM[230.4(1.1)] and Medical Research and Ethics Committee (MREC) of Ministry of Health Malaysia (NMRR-10-798-6522).

\section{RESULTS}

A total of 137 patients were included for this study. After data exploration, four samples out of 137 samples were excluded from analysis. These four samples were having very high warfarin doses (6mg or more) and their residual values were more than three. The four samples excluded were two AVR and two MVR. The two AVR samples excluded were two Indian male and the two MVR samples excluded were one Malay male and one Malay female. Among 133 patients selected for analysis. A total of 59 $(44.4 \%)$ patients were female and $74(55.6 \%)$ were male. Around $61(45.9 \%)$ of them were atrial fibrillation patients, $28(21.1 \%)$ had undergone aortic valve replacement and 44 (33.1\%) had undergone mitral valve replacement. For the races composition, Chinese were the majority with 62 patients $(46.6 \%)$, followed by Malay 56 patients $(42.1 \%)$ and Indian 15 patients $(11.3 \%)$. The mean age of the selected samples was 53.69 years with the standard deviation 15.52 (Table 1). Mean dose (SD) of warfarin for AF patients remained steady with a dose of near $3 \mathrm{mg}(2.83 \mathrm{mg}$ $(0.81)$ in the first week to $2.98 \mathrm{mg}(0.93)$ in the twelfth week) throughout the first 3 months of therapy. Mean dose (SD) of warfarin for AVR patients steadily increased from $2.14 \mathrm{mg}(0.90)$ at first week of warfarin therapy to $2.96 \mathrm{mg}(0.89)$ at twelfth weeks of therapy. Mean dose (SD) of warfarin for AVR patients steadily increased from $2.14 \mathrm{mg}(0.90)$ at first week of warfarin therapy to $2.96 \mathrm{mg}(0.89)$ at twelfth weeks of therapy. 
Table 1: Characteristics of patients selected as samples for the study $(\mathrm{n}=133)$.

\begin{tabular}{ll}
\multicolumn{1}{c}{ Patient's Characteristic } & n (\%) \\
\hline Gender & \\
Female & $59(44.4)$ \\
Male & $74(55.6)$ \\
\hline Indication & \\
Atrial Fibrillation & $61(45.9)$ \\
Aortic Valve Replacement & $28(21.1)$ \\
Mitral Valve Replacement & $44(33.0)$ \\
\hline Race & \\
Malay & $56(42.1)$ \\
Chinese & $62(46.6)$ \\
Indian & $15(11.3)$ \\
\hline
\end{tabular}

For comparison of warfarin doses in initiation phase among patients with aortic valve replacement and mitral valve replacement (within group), the Mauchly's test of sphericity is significant ( $p$-value <0.05) which means the assumption of compound symmetry was not met. Therefore, epsilon correction was used. By using epsilon correction, within group's difference was found significant ( $\mathrm{p}$-value $<0.05$ ). Multiple paired T test was carried out for all possible pairs in three different groups (AF, AVR and MVR). A total of 66 pairs for each group were compared. Bonferroni correction was performed due to the multiple paired comparisons. Since there are 66 comparisons for each group, significance level for each comparison was set at 0.05 / $66=0.0008$. Therefore, $\mathrm{p}$-value that $<0.001$ was considered significant. From multiple paired $\mathrm{T}$ test, within group differences were found significant ( $\mathrm{p}$-value $<0.001$ after bonferroni correction) in AVR group and MVR group. There was no significant different within group in the AF group (Table 2).

Table 2: Comparison of warfarin doses within each group (indication) $(\mathrm{n}=133)$

\begin{tabular}{|c|c|c|c|c|c|c|}
\hline \multirow[t]{2}{*}{ Week } & \multicolumn{2}{|c|}{$\begin{array}{c}\text { Atrial } \\
\text { Fibrillation }\end{array}$} & \multicolumn{2}{|c|}{$\begin{array}{l}\text { Aortic valve } \\
\text { replacement }\end{array}$} & \multicolumn{2}{|c|}{$\begin{array}{l}\text { Mitral valve } \\
\text { replacement }\end{array}$} \\
\hline & $\begin{array}{c}\text { Mean } \\
\text { Diff. }\end{array}$ & $\begin{array}{c}\mathrm{P}- \\
\text { value }\end{array}$ & $\begin{array}{l}\text { Mean } \\
\text { Diff. }\end{array}$ & $\begin{array}{c}\mathbf{P}- \\
\text { value }\end{array}$ & $\begin{array}{l}\text { Mean } \\
\text { Diff. }\end{array}$ & $\begin{array}{c}\mathbf{P}- \\
\text { value }\end{array}$ \\
\hline $1^{\text {st }}-2^{\text {nd }}$ & -0.066 & 0.209 & -0.071 & 0.293 & 0.011 & 0.878 \\
\hline $1^{\mathrm{st}}-3^{\mathrm{rd}}$ & -0.082 & 0.221 & -0.089 & 0.379 & 0.045 & 0.682 \\
\hline $1^{\text {st }}-4^{\text {th }}$ & -0.115 & 0.123 & -0.054 & 0.656 & 0.045 & 0.675 \\
\hline $1^{\mathrm{st}}-5^{\mathrm{th}}$ & -0.172 & 0.030 & -0.268 & 0.061 & 0.045 & 0.694 \\
\hline $1^{\mathrm{st}}-6^{\mathrm{th}}$ & -0.156 & 0.066 & -0.464 & 0.003 & -0.091 & 0.508 \\
\hline $1^{\text {st }}-7^{\text {th }}$ & -0.164 & 0.058 & -0.571 & 0.002 & -0.136 & 0.333 \\
\hline $1^{\text {st }}-8^{\text {th }}$ & -0.148 & 0.109 & -0.625 & 0.001 & -0.216 & 0.128 \\
\hline $1^{\text {st }}-9^{\text {th }}$ & -0.148 & 0.138 & -0.710 & $<0.001$ & -0.250 & 0.088 \\
\hline $1^{\mathrm{st}}-10^{\mathrm{th}}$ & -0.148 & 0.124 & -0.768 & $<0.001$ & -0.284 & 0.038 \\
\hline $1^{\mathrm{st}}-11^{\text {th }}$ & -0.139 & 0.145 & -0.786 & $<0.001$ & -0.364 & 0.010 \\
\hline $1^{\mathrm{st}}-12^{\mathrm{th}}$ & -0.148 & 0.132 & -0.821 & $<0.001$ & -0.466 & 0.001 \\
\hline $2^{\text {nd }}-3^{\text {rd }}$ & -0.016 & 0.621 & -0.018 & 0.713 & 0.034 & 0.660 \\
\hline $2^{\text {nd }}-4^{\text {th }}$ & -0.049 & 0.242 & 0.018 & 0.839 & 0.034 & 0.706 \\
\hline $2^{\text {nd }}-5^{\text {th }}$ & -0.107 & 0.036 & -0.196 & 0.102 & 0.034 & 0.730 \\
\hline $2^{\text {nd }}-6^{\text {th }}$ & -0.090 & 0.117 & -0.393 & 0.005 & -0.102 & 0.335 \\
\hline $2^{\text {nd }}-7^{\text {th }}$ & -0.098 & 0.109 & -0.500 & 0.003 & -0.148 & 0.199 \\
\hline $2^{\text {nd }}-8^{\text {th }}$ & -0.082 & 0.221 & -0.554 & 0.001 & -0.227 & 0.053 \\
\hline $2^{\text {nd }}-9^{\text {th }}$ & -0.082 & 0.261 & -0.643 & 0.001 & -0.261 & 0.033 \\
\hline $2^{\text {nd }}-10^{\text {th }}$ & -0.082 & 0.255 & -0.696 & $<0.001$ & -0.295 & 0.008 \\
\hline $2^{\text {nd }}-11^{\text {th }}$ & -0.074 & 0.303 & -0.714 & $<0.001$ & -0.375 & 0.001 \\
\hline $2^{\text {nd }}-12^{\text {th }}$ & -0.082 & 0.267 & -0.750 & $<0.001$ & -0.477 & $<0.001$ \\
\hline $3^{\text {rd }}-4^{\text {th }}$ & -0.033 & 0.209 & 0.036 & 0.602 & 0.000 & 1.000 \\
\hline $3^{\text {rd }}-5^{\text {th }}$ & -0.090 & 0.015 & -0.179 & 0.086 & 0.000 & 1.000 \\
\hline $3^{\text {rd }}-6^{\text {th }}$ & -0.074 & 0.107 & -0.375 & 0.004 & -0.136 & 0.044 \\
\hline $3^{\text {rd }}-7^{\text {th }}$ & -0.082 & 0.105 & -0.482 & 0.002 & -0.182 & 0.025 \\
\hline $3^{\text {rd }}-8^{\text {th }}$ & -0.066 & 0.251 & -0.536 & 0.001 & -0.261 & 0.002 \\
\hline $3^{\text {rd }}-9^{\text {th }}$ & -0.066 & 0.289 & -0.625 & $<0.001$ & -0.295 & 0.001 \\
\hline $3^{\text {rd }}-10^{\text {th }}$ & -0.066 & 0.289 & -0.679 & $<0.001$ & -0.330 & $<0.001$ \\
\hline
\end{tabular}

\begin{tabular}{|c|c|c|c|c|c|c|}
\hline $3^{\text {rd }}-11^{\text {th }}$ & -0.057 & 0.349 & -0.696 & $<0.001$ & -0.409 & $<0.001$ \\
\hline $3^{\text {rd }}-12^{\text {th }}$ & -0.066 & 0.306 & -0.732 & $<0.001$ & -0.511 & $<0.001$ \\
\hline $4^{\text {th }}-5^{\text {th }}$ & -0.057 & 0.034 & -0.214 & $<0.001$ & 0.000 & 1.000 \\
\hline $4^{\text {th }}-6^{\text {th }}$ & -0.041 & 0.255 & -0.411 & $<0.001$ & -0.136 & 0.038 \\
\hline $4^{\text {th }}-7^{\text {th }}$ & -0.049 & 0.242 & -0.518 & $<0.001$ & -0.182 & 0.025 \\
\hline $4^{\text {th }}-8^{\text {th }}$ & -0.033 & 0.497 & -0.571 & $<0.001$ & -0.261 & 0.002 \\
\hline $4^{\text {th }}-9^{\text {th }}$ & -0.033 & 0.532 & -0.661 & $<0.001$ & -0.295 & 0.001 \\
\hline $4^{\text {th }}-10^{\text {th }}$ & -0.033 & 0.532 & -0.714 & $<0.001$ & -0.330 & $<0.001$ \\
\hline $4^{\text {th }}-11^{\text {th }}$ & -0.025 & 0.635 & -0.732 & $<0.001$ & -0.409 & $<0.001$ \\
\hline $4^{\text {th }}-12^{\text {th }}$ & -0.033 & 0.542 & -0.768 & $<0.001$ & -0.511 & $<0.001$ \\
\hline $5^{\text {th }}-6^{\text {th }}$ & 0.016 & 0.484 & -0.196 & 0.003 & -0.136 & 0.013 \\
\hline $5^{\text {th }}-7^{\text {th }}$ & 0.008 & 0.784 & -0.304 & 0.002 & -0.182 & 0.010 \\
\hline $5^{\text {th }}-8^{\text {th }}$ & 0.025 & 0.517 & -0.357 & 0.001 & -0.261 & 0.001 \\
\hline $5^{\text {th }}-9^{\text {th }}$ & 0.025 & 0.568 & -0.446 & $<0.001$ & -0.295 & 0.001 \\
\hline $5^{\text {th }}-10^{\text {th }}$ & 0.025 & 0.568 & -0.500 & $<0.001$ & -0.330 & $<0.001$ \\
\hline $5^{\text {th }}-11^{\text {th }}$ & 0.033 & 0.454 & -0.518 & $<0.001$ & -0.409 & $<0.001$ \\
\hline $5^{\text {th }}-12^{\text {th }}$ & 0.025 & 0.594 & -0.554 & $<0.001$ & -0.511 & $<0.001$ \\
\hline $6^{\text {th }}-7^{\text {th }}$ & -0.008 & 0.658 & -0.107 & 0.110 & -0.045 & 0.160 \\
\hline $6^{\text {th }}-8^{\text {th }}$ & 0.008 & 0.766 & -0.161 & 0.026 & -0.125 & 0.015 \\
\hline $6^{\text {th }}-9^{\text {th }}$ & 0.008 & 0.811 & -0.250 & 0.011 & -0.159 & 0.015 \\
\hline $6^{\text {th }}-10^{\text {th }}$ & 0.008 & 0.821 & -0.304 & 0.002 & -0.193 & 0.006 \\
\hline $6^{\text {th }}-11^{\text {th }}$ & 0.016 & 0.658 & -0.321 & 0.001 & -0.273 & $<0.001$ \\
\hline $6^{\text {th }}-12^{\text {th }}$ & 0.008 & 0.837 & -0.357 & 0.001 & -0.375 & $<0.001$ \\
\hline $7^{\text {th }}-8^{\text {th }}$ & 0.016 & 0.419 & -0.054 & 0.083 & -0.080 & 0.033 \\
\hline $7^{\text {th }}-9^{\text {th }}$ & 0.016 & 0.597 & -0.143 & 0.030 & -0.114 & 0.031 \\
\hline $7^{\text {th }}-10^{\text {th }}$ & 0.016 & 0.621 & -0.196 & 0.005 & -0.148 & 0.014 \\
\hline $7^{\text {th }}-11^{\text {th }}$ & 0.025 & 0.471 & -0.214 & 0.005 & -0.227 & 0.001 \\
\hline $7^{\text {th }}-12^{\text {th }}$ & 0.016 & 0.641 & -0.250 & 0.002 & -0.330 & $<0.001$ \\
\hline $8^{\text {th }}-9^{\text {th }}$ & 0.000 & 1.000 & -0.089 & 0.134 & -0.034 & 0.183 \\
\hline $8^{\text {th }}-10^{\text {th }}$ & 0.000 & 1.000 & -0.143 & 0.030 & -0.068 & 0.083 \\
\hline $8^{\text {th }}-11^{\text {th }}$ & 0.008 & 0.766 & -0.161 & 0.026 & -0.148 & 0.001 \\
\hline $8^{\text {th }}-12^{\text {th }}$ & 0.000 & 1.000 & -0.196 & 0.009 & -0.250 & $<0.001$ \\
\hline $9^{\text {th }}-10^{\text {th }}$ & 0.000 & 1.000 & -0.054 & 0.083 & -0.034 & 0.262 \\
\hline $9^{\text {th }}-11^{\text {th }}$ & 0.008 & 0.568 & -0.071 & 0.043 & -0.114 & 0.006 \\
\hline $9^{\text {th }}-12^{\text {th }}$ & 0.000 & 1.000 & -0.107 & 0.011 & -0.216 & 0.001 \\
\hline $10^{\text {th }}-11^{\text {th }}$ & 0.008 & 0.321 & -0.018 & 0.326 & -0.080 & 0.007 \\
\hline $10^{\text {th }}-12^{\text {th }}$ & 0.000 & 1.000 & -0.054 & 0.083 & -0.182 & 0.002 \\
\hline $11^{\text {th }}-12^{\text {th }}$ & -0.008 & 0.321 & -0.036 & 0.161 & -0.102 & 0.048 \\
\hline
\end{tabular}

*multiple paired $t$ test with 66 comparisons

$*$ Bonferroni correction applied, significance level $=0.05$ / $66=0.0008$

For comparison of warfarin doses in initiation phase between patients with AF, AVR and MVR, analysis were performed using one-way repeated measures ANOVA and multiway repeated measures ANCOVA. Both analyses showed that the dose of warfarin was significantly different for AVR and AF in the first 5 weeks by comparing their $95 \%$ confidence interval respectively after controlled age, gender and race. In the first 5 weeks, the upper limit and lower limit of AVR group did not overlap with upper limit and lower limit of AF group (Table 3).

In one-way repeated measures ANOVA, indications (AF, AVR and MVR) were found not significantly different in warfarin doses regardless of time with the p-value 0.143. Post-hoc test showed that the mean difference between these 3 indications are not significant ( $\mathrm{p}$-value >0.05) (Table 4). However, Indications (AF, AVR and MVR) were found significantly different in warfarin doses regardless of time with the $p$-value 0.036 when age, gender and race were controlled using multi-way repeated measures ANCOVA. Post-hoc test was carried out by comparing the estimated marginal means with using Sidak method to adjust for multiple comparisons. Sidak method was chosen because we assumed that the individual tests are independent. The significant difference of warfarin doses occurred between AF group and AVR group with the p-value of 0.03 . 
Table 3: Comparison of warfarin doses among three different indication between one-way repeated measures ANOVA and multi-way repeated measures ANCOVA $(\mathrm{n}=133)$.

\begin{tabular}{|c|c|c|c|c|c|}
\hline \multirow{2}{*}{$\begin{array}{c}\text { Time } \\
\text { (week) }\end{array}$} & \multirow{2}{*}{ Groups } & \multicolumn{2}{|c|}{ One-way repeated measures ANOVA } & \multicolumn{2}{|c|}{ Multi-way repeated measures ANCOVA } \\
\hline & & Mean warfarin doses (mg) & $95 \% \mathrm{CI}$ & Mean warfarin doses (mg) & $95 \% \mathrm{CI}$ \\
\hline \multirow[t]{3}{*}{$1^{\mathrm{st}}$} & Atrial Fibrillation & 2.83 & $2.60,3.05$ & 2.99 & $2.71,3.27$ \\
\hline & Aortic valve replacement & 2.14 & $1.81,2.48$ & 2.13 & $1.75,2.51$ \\
\hline & Mitral valve replacement & 2.60 & $2.34,2.87$ & 2.74 & $2.41,3.07$ \\
\hline \multirow[t]{3}{*}{$2^{\text {nd }}$} & Atrial Fibrillation & 2.89 & $2.67,3.12$ & 3.10 & $2.83,3.37$ \\
\hline & Aortic valve replacement & 2.21 & $1.89,2.54$ & 2.20 & $1.82,2.57$ \\
\hline & Mitral valve replacement & 2.59 & $2.33,2.85$ & 2.73 & $2.41,3.05$ \\
\hline \multirow[t]{3}{*}{$3^{\text {rd }}$} & Atrial Fibrillation & 2.91 & $2.68,3.14$ & 3.13 & $2.86,3.40$ \\
\hline & Aortic valve replacement & 2.23 & $1.90,2.57$ & 2.24 & $1.87,2.62$ \\
\hline & Mitral valve replacement & 2.56 & $2.29,2.82$ & 2.73 & $2.41,3.05$ \\
\hline \multirow[t]{3}{*}{$4^{\text {th }}$} & Atrial Fibrillation & 2.94 & $2.72,3.17$ & 3.17 & $2.90,3.44$ \\
\hline & Aortic valve replacement & 2.20 & $1.87,2.53$ & 2.16 & $1.79,2.53$ \\
\hline & Mitral valve replacement & 2.56 & $2.29,2.82$ & 2.70 & $2.38,3.02$ \\
\hline \multirow[t]{3}{*}{$5^{\text {th }}$} & Atrial Fibrillation & 3.00 & $2.78,3.22$ & 3.20 & $2.93,3.47$ \\
\hline & Aortic valve replacement & 2.41 & $2.08,2.74$ & 2.43 & $2.05,2.80$ \\
\hline & Mitral valve replacement & 2.56 & $2.29,2.82$ & 2.73 & $2.40,3.05$ \\
\hline \multirow[t]{3}{*}{$6^{\text {th }}$} & Atrial Fibrillation & 2.98 & $2.76,3.21$ & 3.15 & $2.87,3.44$ \\
\hline & Aortic valve replacement & 2.61 & $2.27,2.95$ & 2.59 & $2.20,2.98$ \\
\hline & Mitral valve replacement & 2.69 & $2.42,2.96$ & 2.88 & $2.54,3.22$ \\
\hline \multirow[t]{3}{*}{$7^{\text {th }}$} & Atrial Fibrillation & 2.99 & $2.75,3.23$ & 3.17 & $2.88,3.47$ \\
\hline & Aortic valve replacement & 2.71 & $2.36,3.07$ & 2.68 & $2.27,3.09$ \\
\hline & Mitral valve replacement & 2.74 & $2.46,3.02$ & 2.91 & $2.55,3.26$ \\
\hline \multirow[t]{3}{*}{$8^{\text {th }}$} & Atrial Fibrillation & 2.98 & $2.73,3.21$ & 3.14 & $2.84,3.45$ \\
\hline & Aortic valve replacement & 2.77 & $2.41,3.13$ & 2.71 & $2.29,3.14$ \\
\hline & Mitral valve replacement & 2.82 & $2.53,3.10$ & 2.98 & $2.61,3.34$ \\
\hline \multirow[t]{3}{*}{$9^{\text {th }}$} & Atrial Fibrillation & 2.98 & $2.73,3.23$ & 3.18 & $2.87,3.49$ \\
\hline & Aortic valve replacement & 2.86 & $2.49,3.23$ & 2.77 & $2.34,3.20$ \\
\hline & Mitral valve replacement & 2.85 & $2.56,3.15$ & 3.00 & $2.63,3.38$ \\
\hline \multirow[t]{3}{*}{$10^{\text {th }}$} & Atrial Fibrillation & 2.98 & $2.73,3.22$ & 3.17 & $2.87,3.48$ \\
\hline & Aortic valve replacement & 2.91 & $2.49,3.23$ & 2.82 & $2.40,3.24$ \\
\hline & Mitral valve replacement & 2.89 & $2.60,3.17$ & 3.02 & $2.66,3.38$ \\
\hline \multirow[t]{3}{*}{$11^{\text {th }}$} & Atrial Fibrillation & 2.97 & $2.72,3.21$ & 3.18 & $2.87,3.48$ \\
\hline & Aortic valve replacement & 2.93 & $2.57,3.29$ & 2.84 & $2.42,3.26$ \\
\hline & Mitral valve replacement & 2.97 & $2.68,3.25$ & 3.09 & $2.72,3.45$ \\
\hline \multirow[t]{3}{*}{$12^{\text {th }}$} & Atrial Fibrillation & 2.98 & $2.73,3.22$ & 3.19 & $2.88,3.51$ \\
\hline & Aortic valve replacement & 2.96 & $2.60,3.33$ & 2.87 & $2.43,3.30$ \\
\hline & Mitral valve replacement & 3.07 & $2.78,3.36$ & 3.16 & $2.79,3.53$ \\
\hline
\end{tabular}

* Age, gender and race were controlled under multi-way repeated measures ANCOVA

Table 4: Comparison of mean difference of warfarin doses among three different indications regardless of time ( $\mathrm{n}=133$ )

\begin{tabular}{|c|c|c|c|c|}
\hline \multirow[b]{2}{*}{ Compare } & \multicolumn{2}{|c|}{ One-way RM ANOVA } & \multicolumn{2}{|c|}{ Multi-way RM ANCOVA $^{\text {b }}$} \\
\hline & $\begin{array}{c}\text { Mean Difference }^{\mathrm{a}} \\
(95 \% \mathrm{CI})\end{array}$ & P-value & $\begin{array}{c}\text { Mean Difference } \\
(95 \% \text { CI })\end{array}$ & P-value \\
\hline Atrial Fibrillation - Aortic valve replacement & $0.373(-0.114,0.859)$ & 0.170 & $0.613(0.045,1.182)$ & 0.030 \\
\hline Atrial Fibrillation - Mitral valve replacement & $0.211(-0.211,0.633)$ & 0.466 & $0.260(-0.270,0.790)$ & 0.555 \\
\hline Aortic valve replacement-Mitral valve replacement & $-0.162(-0.677,0.354)$ & 0.740 & $-0.353(-0.239,0.946)$ & 0.389 \\
\hline
\end{tabular}

${ }^{\mathrm{a}} \mathrm{S} c h e f f e$ test was used as post-hoc test

${ }^{\mathrm{b}}$ Age, gender and race were controlled

${ }^{\mathrm{c}}$ Adjustment for multiple comparisons: Sidak

\section{DISCUSSION}

The mean warfarin dose in this study was lower than the results from Meijer et al where they reported the mean warfarin dose of warfarin for mechanical valve replacement patients steadily increased from $3.79 \mathrm{mg}$ to $4.37 \mathrm{mg}$ at first week and thirteenth week respectively (Meijer et al., 2009). Similarly, the mean warfarin dose in the study also lower as compared to results from Mesbah Rahman et al where they reported the mean dose
(SD) of warfarin increased from $3.55 \mathrm{mg}$ (1.94) to $5.09 \mathrm{mg}$ (2.03) at the first week and twelfth week respectively after heart valve replacement (Rahman et al., 2006). The trend of lower mean warfarin dose in the study compared to the one reported in Western countries (Meijer et al., 2009; Rahman et al., 2006) was consistent with studies done by others Asian countries which showed Asian populations generally need lower dose of warfarin to achieved INR target (Jonas and McLeod, 2009; Mori et al., 2002; Sun et al., 2003; Yang et al., 2010; Zhou et al., 2005). This 
might due to genetic difference between Asian and Western population where Vitamin $\mathrm{K}$ epoxide reductase complex subunit 1 (VKORC1) genotype (known to affect warfarin sensitivity (Jonas and McLeod, 2009)) frequencies of Asian and Caucasian patients were different (Yang et al., 2010). Besides, diet which was different between Asian and Western populations might also contributed to the difference in warfarin dose needed (Jonas and McLeod, 2009)

The mean warfarin dose in AVR and MVR patients after 3 months of therapy was $38.3 \%$ and $18.1 \%$ higher than first week of therapy. The result was consistent with study done by Mesbah Rahman et al and Meijer et al. In result reported by Mesbah Rahman et al, the dose requirement of warfarin after 3 months of therapy was $43 \%$ higher compared to first week of therapy (Rahman et al., 2006). For Meijer et al, the dose requirement of warfarin after 3 months of therapy was $26 \%$ higher compared to first week of therapy (Meijer et al., 2009). However, Mesbah Rahman et al did not separate the dose increment in AVR and MVR group. Besides, Meijer et al did not include patients with MVR.

Results showed that warfarin requirements were in increasing trend during the first three months after heart valve replacement (both AVR and MVR) but this pattern was absent in patients with AF. The trend was consistent with previous studies (Meijer et al., 2009; Rahman et al., 2006). Several factors might contribute to the increasing trend of mean warfarin dose among patients with AVR and MVR. Factors known to affect the warfarin sensitivity including recovery from hypoalbuminemia after surgery (Ageno and Turpie, 1999; Rahman et al., 2006), improvement in hepatic congestion and cardiac output (Rahman et al., 2006), increase in dietary intake of vitamin $\mathrm{K}$ and decreasing adherence to therapy (Meijer et al., 2009).

Hypoalbuminemia can affect the volume of distribution and half-life of warfarin since warfarin was almost $99 \%$ bound to albumin (Charles F. Lacy et. al, 2009). Hypoalbuminemia enhance the response of warfarin in postoperative cardiac surgical patients (Ageno and Turpie, 1999).

We could not trace the patients' serum albumin level in this study. However, except patients with some underlying diseases like persistent sepsis, hepatic impairment, nephrotic syndrome or hyperthyroidism that decreased synthesis, affect redistribution and increased loss or catabolism of albumin (Crook, 2009); it is unlikely that hypoalbuminemia levels would be persistent throughout the 3 months period. When patients recover from heart valve surgery, the improvement in albumin level would cause increment of the warfarin dose requirement in order to maintain the targeted INR. Nevertheless, it seems unlikely that the albumin level would steadily continue to increase over a period of three months (Meijer et al., 2009). Therefore, improvement of albumin level might be the factor that caused the need to increase warfarin dose until patient achieved normal albumin level but not the increasing trend throughout the period of three months.

Among 3 different indication (AF, AVR and MVR), the dose of warfarin was significantly different for AVR and AF in the first 5 weeks. This might due to physiological changes after heart valve surgery as discussed before.

The targeted INR set for patients with AF, AVR and MVR was 2.0 - 3.0, 1.8 - 2.5 and 2.5 - 3.5 respectively in Hospital Penang. This might explain why there was no significant difference in the dose throughout the 3 months study period between patients with AF and MVR. Hypoalbuminemia, hepatic congestion and decreased in cardiac output might also cause the need of lower warfarin dose in MVR patients same as AVR patients in the early phase of post-surgery. However, as the targeted INR for MVR patients was higher compared to AVR patients, therefore a higher warfarin dose needed for MVR patients to achieve the higher targeted INR compared to AVR patients. As a result, there was no significant difference between mean warfarin dose in AF and MVR patients.

Our study was limited by retrospective study design. A few factors that found to be affecting warfarin doses could not be measured in our study as it was not recorded in patients' record. These included body mass index (BMI), body weight, valve type and compliance to medications. The unmeasured variables may have impact on warfarin doses in initial phase of warfarin therapy. Ratio composition for indications was not equal in our study. AVR patients were least recruited to this study due to lack of patients. Lack of samples for AVR patients may reduce the power of the study.

Since warfarin doses was in increasing trend and the dose was significantly different during the first three months of therapy in AVR and MVR patients, monitoring during the initial 3 months period should be frequent in these group of patients. It is likely that AVR and MVR patients require an increase in the warfarin dose in order to maintain the targeted INR to avoid thromboembolism event.

\section{CONCLUSION}

As conclusion, heart valve replacement patients (AVR or MVR) showed increasing trend in warfarin doses during the initial phase of warfarin therapy. This trend was not observed in AF patients. The dose of warfarin was significantly different within first 12 weeks of warfarin initiation for AVR and MVR group but there was no significant difference in AF group. The dose of warfarin was significantly different among AVR and AF patients for first 5 weeks of warfarin initiation. Indication was found to be significantly affecting warfarin doses in the initial phase of warfarin therapy.

\section{Financial support and sponsorship: Nil.}

Conflict of Interests: There are no conflicts of interest.

\section{REFERENCES}

Ageno W, Turpie AG. Exaggerated initial response to warfarin following heart valve replacement. Am J Cardiol, 1999; 84(8): 905-908.

Charles FL, et al. 2009. Drug Information Handbook $18^{\text {th }}$ Editioned. Lexi-Comp 
Crook MA. Hypoalbuminemia: the importance of correct interpretation. Nutrition, 2009; 25(10): 1004-1005.

Douketis JD, Foster GA, Crowther MA, Prins MH, Ginsberg JS. Clinical risk factors and timing of recurrent venous thromboembolism during the initial 3 months of anticoagulant therapy. Arch Intern Med, 2000; 160(22): 3431-3436.

Fuster V, et al. ACC/AHA/ESC Guidelines for the Management of Patients With Atrial Fibrillation: Executive Summary A Report of the American College of Cardiology/American Heart Association Task Force on Practice Guidelines and the European Society of Cardiology Committee for Practice Guidelines and Policy Conferences (Committee to Develop Guidelines for the Management of Patients With Atrial Fibrillation) Developed in Collaboration With the North American Society of Pacing and Electrophysiology. Circulation, 2001; 104(17): 2118-2150.

Haines ST, Witt DM, Nutescu EA. 2008. Pharmacotherapy: a pathophysiologic approach. In: Dipiro JT, Talbert RL, Yee GC, Matzke GR. (eds.), Venous Thromboembolism 7th ed. New York, Chicago, San Francisco, Lisbon, London: McGrawHill 331-372.

Hall AM, Wilkins MR. Warfarin: a case history in pharmacogenetics. Heart, 2005; 91(5): 563-564.

Jonas DE, McLeod HL. Genetic and clinical factors relating to warfarin dosing. Trends in Pharmacological Sciences, 2009; 30(7): 375386.

Kulik A, et al. Early postoperative anticoagulation after mechanical valve replacement: a systematic review. Ann Thorac Surg, 2006; 81(2): 770-781.

Lee VW, et al. Factors affecting the maintenance stable warfarin dosage in Hong Kong Chinese patients. J Thromb Thrombolysis, 2005; 20(1): 33-38.

Meijer K, Kim YK, Schulman S. Decreasing warfarin sensitivity during the first three months after heart valve surgery: implications for dosing. Thromb Res, 2009; 125(3): 224-229.

Mori $\mathrm{T}$, et al. Anticoagulant therapy after prosthetic valve replacement -optimal PT-INR in Japanese patients. Ann Thorac Cardiovasc Surg, 2002; 8(2): 83-87.

Rahman M, BinEsmael TM, Payne N, Butchart EG. Increased sensitivity to warfarin after heart valve replacement. Ann Pharmacother, 2006; 40(3): 397-401.
Salem DN, O'Gara PT, Madias C, Pauker SG. Valvular and structural heart disease: American College of Chest Physicians EvidenceBased Clinical Practice Guidelines (8th Edition). Chest, 2008; $133(6$ Suppl): 593S-629S.

Sameerah SAR, Sarojini S. 2007. Malaysian Statistics on Medicine 2005. Ministry of Health Malaysia.

Sun X, Hu S, Qi G, Zhou Y. Low standard oral anticoagulation therapy for Chinese patients with St. Jude mechanical heart valves. Chin Med J (Engl), 2003; 116(8): 1175-1178.

Takahashi H, Wilkinsin G, Caraco Y. Population differences in S- warfarin metabolism between CYP2C9 genotype-matched Caucasion and Japanese patients. Clin Pharmacol Ther, 2003; 73: 253-263.

Wadelius $\mathrm{M}$, et al. Warfarin sensitivity related to CYP2C9, CYP3A5, ABCB1 (MDR1) and other factors. The Pharmacogenomics Journal, 2004; 4(1): 40-48.

Yang L, Ge W, Yu F, Zhu H. Impact of VKORC1 gene polymorphism on interindividual and interethnic warfarin dosage requirement--a systematic review and meta analysis. Thromb Res, 2010; 125(4): e159-166.

Zhou XM, et al. Low-dose anticoagulation in Chinese patients with mechanical heart valves. Asian Cardiovasc Thorac Ann, 2005; 13(4): 341-344

\section{How to cite this article:}

Teoh CJ, Aniza AA, Wan-Arfah B, Naing NN. The Initiation Phase of Warfarin Therapy: Differences of Dose among Patients with Aortic Valve Replacement, Mitral Valve Replacement and Atrial Fibrillation. J App Pharm Sci, 2016; 6 (11): 028-033. 